\title{
RECOMMENDATIONS FOR INFECTION CONTROL IN DENTAL LABORATORIES DURING COVID-19 PANDEMIC AND NORMALIZATION PERIOD
}

\section{Fulya Basmaci ${ }^{1}$, Mehmet Ali Kilicarslan², Figen Cizmeci Senel ${ }^{3}$}

\author{
${ }^{1}$ Ankara Yildirim Beyazıt University, Faculty of Dentistry, Department of Prosthodontics, Ankara, Turkey. \\ 2 Ankara University, Faculty of Dentistry, Department of Prosthodontics, Ankara, Turkey. \\ ${ }^{3}$ Ministry of Health, Health Institutes of Turkey, Ankara, Turkey
}

\author{
Address for Correspondence: Assist. Prof. Fulya Basmaci E-mail: fulyacizmeci@hotmail.com \\ Received: 17.01.2021; Accepted: 16.03.2021; Available Online Date: 27.05.2021 \\ (C) Copyright 2021 by Dokuz Eylül University, Institute of Health Sciences - Available online at https://dergipark.org.tr/en/pub/jbachs
}

Cite this article as: Basmaci F, Kilicarslan MA, Cizmeci-Senel F. Recommendations for Infection Control in Dental Laboratories During Covid-19 Pandemic and Normalization Period. J Basic Clin Health Sci 2021; 2: 175-180.

\begin{abstract}
Coronavirus disease 2019 (Covid-19), a novel human coronavirus (SARS-CoV-2) causing a severe acute respiratory syndrome, was first reported in Wuhan, China at the end of 2019. SARS-CoV-2 is transmitted primarily between people through respiratory droplets. Direct contact with blood, oral fluids and other patient materials has been reported as another transmission route of Covid-19. Dental impressions, trays, occlusal records, prostheses or appliances can be contaminated with bacteria, viruses and fungi. The necessity of determining the precautions for safety in dental laboratories, one of the dental service components, and evaluating them periodically due to their potential as a source of cross-infection has come into prominence during the Covid-19 pandemic. For this purpose, we publish this guideline for dental laboratories.
\end{abstract}

Keywords: COVID-19, dental impression, dental laboratory, dental prosthesis, Sars-CoV-2

The new coronavirus disease (COVID-19) was first identified in Wuhan, China in December 2019, and declared as a global pandemic by the World Health Organization on 11 March 2020. $(1,2,3)$

Coronavirus, known as the cause of COVID-19, is spread mainly from person-to-person through respiratory droplets. The disease can also be transmitted by touching the oral, nasal or eye mucous membranes with dirty hands that come into contact with the surfaces contaminated by droplets from an infected person's coughs or sneezes. Direct contact with blood, oral fluids and other patient materials has been reported as another transmission route of Covid-19. High-speed rotary instruments used in routine dentistry practices and aerosols released into the air increase the risk of transmission of coronavirus. Therefore, dental procedures are considered risky in this regard. SARS-CoV-2 has been identified in the saliva of infected patients, so dental laboratory staff is also at risk of Covid-19 infection. $(4,5,6,7,8)$

Dental impressions, trays, occlusal records, models, mock-ups, appliances and prostheses (prosthetic supplies) can be contaminated with bacteria, viruses and fungi. The necessity of determining the precautions for safety in dental laboratories, one of the dental service components, and evaluating them periodically due to their potential as a source of crossinfection has come into prominence during the Covid19 pandemic. For this purpose, we have prepared a guideline for dental laboratories due to the lack of a 
specific guideline for dental laboratories in the literature.

Laboratory services can be outsourced or offered inhouse for healthcare facilities. Standard infection control measures should be followed to protect infections in these units. In this pandemic process, all prosthetic supplies should be treated like COVID-19 case materials.

All institutions, organizations, units, sub-units and persons should prepare to fulfill their roles and responsibilities for gradual return to normal life during the COVID-19 pandemic.

\section{CONTROL MEASURES FOR PERSONNEL}

All laboratory staff should take particular care of hand hygiene. It is recommended to wash hands with soap and water for at least 20 seconds to ensure hand hygiene. If soap and water are not readily available, an alcohol-based hand sanitizer that contains at least $70 \%$ alcohol can be used. For this purpose, hand antiseptics should be placed in suitable areas in the laboratory. $(5,6)$

- Performing hand hygiene before and after all kinds of dental impression, model and prosthesis contact is especially important for removing pathogens that might have been transferred to bare hands before putting on and removing personal protective equipment (PPE), including gloves.

- Laboratory staff should wear uniforms in both disinfection area and laboratory workspace, and also should wear box apron, medical mask, protective glasses/face shields and gloves.

- Mobile electronic devices should not be taken into the disinfection area/room. The devices should be kept in other areas where they cannot be easily contaminated or act as a carrier object for infection. These devices should be covered by a physical barrier or decontaminated before leaving the laboratory.

- PPE should not be worn outside the laboratory.

- All laboratory staff should have daily fever measurements before entering the laboratory and should be questioned for Covid-19 symptoms.

- All laboratory staff should be informed periodically about Covid-19 symptoms and prevention methods, and safety symbols and hazard signs containing this information should be placed in visible locations of the laboratory.

- Training and information materials provided to laboratory staff should be compatible with the guidelines and other informative materials prepared and published by the Ministry of Health.

- All laboratory staff should watch for and report the signs and symptoms of COVID-19 infection in both themselves and their families.

- If a laboratory employee is tested positive for Covid-19, the management of other personnel should be planned by the relevant department according to the algorithm of the Assessment of Health Workers Contact with Covid-19. $(3,5,6,7,9)$

\section{CONTROL MEASURES FOR LABORATORY}

\section{PHYSICAL CONDITIONS}

\section{Work Area and Other Units}

- Laboratories should be designed in accordance with certification standards; and should not involve in procedures that do not comply with their qualifications.

- Work methods and patterns should be reviewed by considering social distance rules.

- Visitors should not be allowed to enter laboratories, except dentists who comes to report work details. Meetings should be held in separate rooms reserved for this purpose by paying attention to social isolation rules.

- Areas such as office or dining hall should be arranged according to social distance rules.

\section{Ventilation and Air Conditioning Systems}

- Laboratory areas without technical equipment such as front desks should be well ventilated by allowing clean air circulation through windows and doors open to fresh air.

- If ventilation systems are used, they should be inspected, maintained, and cleaned regularly. Air conditioning systems with $100 \%$ fresh air intake unit can absorb and discharge virus particles floating indoors. Indoor air is replaced with clean and filtered fresh outdoor air and droplets suspended in the air get diluted. Thus, the risk of airborne transmission is reduced.

- As air conditioning systems with $100 \%$ indoor air circulation (Fan Coil Unit, Split air conditioner etc.) constantly circulate the same air, they increase the risk of respiratory infections by 
leading to an increase in the density of microorganisms such as viruses and bacteria in closed spaces over time. Therefore, these devices should not be used during the pandemic.

- Ceiling or portable fans that significantly blow room air and cause air flow should not be used.

- Vacuum pumps should be used to provide air intake in contour areas, where employees should use (FFP2/FFP3) industrial dust masks in accordance with TS EN 149 Standard. $(4,6,7,9)$

\section{Ambient and Surface Cleaning}

- All areas should be cleaned frequently and disinfected regularly. A particular attention should be paid to frequently contacted surfaces (electrical switches, door handles, chair arms, etc.) during cleaning and disinfection. Cleaning should be carried out using water and detergent starting from clean areas to dirty ones.

a. Both floor and surface disinfections should be performed using 1/100 diluted sodium hypochlorite (household bleach).

b. Surfaces contaminated with patient secretion should be disinfected using $1 / 10$ diluted sodium hypochlorite (household bleach) or chlorine tablets (according to product recommendation). 70\% alcohol can also be used for this purpose. The surface(s) being disinfected should remain wetted for at least 1 minute.

- Common use devices such as phones and computer keyboards should be covered with transparent protectors and disinfected after each use.

- Toilets should be cleaned frequently and disinfected regularly. Liquid soap and paper towels should always be available in toilets. Air hand dryers should not be used. 5,6

\section{GENERAL STERILIZATION / DISINFECTION RULES}

- The communication of a patient, dentist and dental laboratories with online methods is recommended.

- A disinfection area separated from offices should be established in the laboratory. All kinds of materials provided from outside without disinfection should be first stored in this area, disinfected without delay, and transferred to work areas only after complete disinfection.
- $\quad$ Liquid-proof disposable covers should be placed under cleaning materials to prevent surface contamination in disinfection areas.

- Blood and saliva of all patients should be considered potentially infectious, and a behavioral model should be developed accordingly.

- If hands or other skin surfaces are contaminated with patient blood or saliva, they should be washed immediately with soap and water.

- At the stage of acceptance of the impressions or prostheses into the laboratory, computer and book entries should be recorded without opening the package in line with the information on the label printed on the upper surface of the package.

- Laboratory staff should wear gloves, masks, aprons and protective glasses/face shield during the acceptance and opening of work packages that are delivered to the laboratory.

- Disposable packaging materials should be discarded; and reusable containers should be disinfected.

- Necessary precautions should be taken to prevent cross-infection of dental materials such as drill heads, burs, relays and discs used for prosthesis construction. These materials should be cleaned in an ultrasonic bath to eliminate debris, and then sterilized in an autoclave. Those that are not suitable for sterilization should be disinfected by immersion method or disposable products should be preferred.

- All brushes, including cotton wheel brushes, should be kept in disinfectant after each use in accordance with the manufacturer's recommendation. UV sterilizers or autoclaves are recommended for sterilization.

- Materials such as pumice should be prepared in small amounts and renewed for each process. It is also recommended to add disinfectant to polishing pumice and use vacuum aspiration systems in order to prevent infections.

- Procedures for patients with confirmed or suspected Covid-19 should be performed under isolation as the last work of the day.

- All wastes that have been in contact with patients' mouth, blood and secretions should be disposed of as medical. $(5,6,7,10,11,12)$ 
III. DURING THE TRANSFER OF DENTAL IMPRESSIONS, MOCK-UPS OR PROSTHESES FROM CLINIC TO LABORATORY AND LABORATORY TO CLINIC

\section{General Rules}

- All prosthetic supplies that have been in contact with patients' oral cavity should be sterilized or disinfected before laboratory studies and use in patients.

- If laboratory services are outsourced, a written procedure for the disinfection of impressions, tryin materials, prostheses, appliances and other supplies should be established between the outsourcing healthcare facility and the laboratory. The responsible party for disinfection should be made clear for each stage of the process starting from impression to the final stage of the prosthesis. Thus, damage to materials is prevented by not repeating the effective disinfection procedures unnecessarily.

- Exit records of all works should be kept, and employees who perform and transfer the works should be recorded properly.

- In order to prevent contamination, the parties responsible for prosthetic construction stages including impression, modeling, try-in and finalization should carry out disinfection and/or sterilization as soon as possible after each procedure.

- All materials used in these stages should be disinfected in accordance with the manufacturer's recommendation to prevent them from undergoing structural change. Disinfectants suitable for these materials and licensed by the Ministry of Health should be used in disinfection procedures.

- Autoclave sterilization is recommended at autoclavable stages and materials.

- Disinfected impressions, prostheses and other materials should be placed in a sealed box or locked pouch, where a label indicating disinfection should be affixed.

- If biological residues such as blood and saliva are detected on incoming works despite the disinfection note, disinfection procedures should be repeated in the laboratory.

- If the institution offers in-house laboratory services, its employees should comply with standard infection control measures and perform the aforementioned procedures for outsourced laboratory services $(13,14,15)$
Disinfection, Transfer and Delivery Protocol of Dental Impressions, Mock-ups, Prostheses and Appliances in Clinic and Laboratory

- After being removed from the patient's mouth, prosthetic supplies should be cleaned from visible blood and saliva under running water without being deformed.

- The best time to clean and disinfect these supplies is defined as the shortest possible time after they are removed from the patient's mouth, without allowing blood, saliva or other biological residues to dry.

- Disinfection of impression materials can be performed by dipping or spraying the materials using various disinfectants at different concentrations and dwell times.

- During the disinfection of impression materials, glutaraldehyde $(0.5 \%, 2 \%, 2.2 \%$ and $2.45 \%)$, sodium hypochlorite $(\mathrm{NaOCl})(0.5 \%, 0.525 \%$, $1 \%, 4 \%$ and $5.25 \%)$, chlorine compounds $(0.2 \%$ chlorhexidine), hydrogen peroxide $(0.5 \%)$, iodophores $(5 \%$ and $10 \%)$, and phenol compounds $(7 \%)$ can be used according to the type of impression material.

- It is known that different impression materials react to different disinfection methods, durations of disinfection, disinfectant types and concentrations. Therefore, the manufacturer's recommendations for impression materials should be followed in determining the proper disinfection method.

- It is recommended to carry out the disinfection of impression materials in accordance with the following material classification:

a) $0.5 \%$ sodium hypochlorite or iodophores can be used for irreversible hydrocolloids (alginate). Disinfection by spraying is recommended, as prolonged immersion can cause imhibition-related deterioration.

b) lodophores or sodium hypochlorite can be used for reversible hydrocolloids (Agar).

c) lodophores and chlorine compounds can be used for polyethers. Gluteraldehyde is not recommended. Disinfection by spraying is recommended, as prolonged immersion can cause distortion.

d) $2 \%$ gluteraldehyde, iodophores and chlorine compounds can be used for disinfection of zinc oxide eugenol.

e) Silicone impression materials can be immersed in disinfectant. Gluteraldehyde, iodophores and chlorine compounds can be used as disinfectants. 
f) lodophores and chlorine compounds can be used for disinfection of removable dentures.

g) It is recommended to autoclave fixed dentures. Gluteraldehyde can be used as a disinfectant.

h) Transfer containers should be washed with plenty of water and detergent, and their surfaces should be disinfected properly.

- If the disinfected impressions are transferred directly from the clinic to the laboratory, they should be placed in a locked pouch or transfer box, where a label indicating disinfection should be affixed.

- If the impressions are not directly transferred to the laboratory (if there are transfer rooms such as those in hospitals or if they are sent to the laboratory after the model is obtained), the intermediate units should label job credentials as fully visible, and the intermediate transfer should be performed using bags or containers to prevent contamination.

- It is recommended to sterilize work models made of autoclavable materials or finishing works in the autoclave and to send autoclave bags with a timestamp to the clinic without deterioration. It is also recommended to take external transfer packages outside the clinic by using an autoclave package.

- All information about patients, physicians, job qualifications and entry-exit dates should be recorded on the registration form without being contaminated; and the form should be transmitted in a way that prevents contact with the model or impression.

- Employees should not touch the package with contaminated hands or gloves. $(10,11,14,16,17,18,19)$

\section{Code of Conduct for Transfer Personnel}

- Laboratory and clinical staff and/or physicians should communicate according to social distancing rules.

- Personnel should not touch impressions or works with bare hand and should wear a mask in the clinic or laboratory.

- Personnel should behave in a way that prevents cross-contamination of frequently used surfaces such as door handles in clinical and laboratory entrances and exits. 5,6

\section{REFERENCES}

1. Hongzhou L, Stratron CW, Tank YW: Outbreak of pneumonia of unknown etiology in Wuhan, China: The mystery and the miracle. J. Med.Virol 2020;92(4):401-402.

2. Chen J: Pathogenicity and transmissibility of 2019-nCoV-A quick overview and comparison with other emerging viruses. Microbes Infect 2020;22(2):69-71.

3. Peng $X, X u X$, Li Y, Cheng L, Zhou X, Ren B: Transmission routes of 2019-nCoV and controls in dental practice. International Journal of Oral Sciences. 2020;12(9):1-6.

4. To KK, Tsang OT, Yip CC et al: Consistent detection of 2019 novel coranavirus in saliva. Clin Infect.Dis. 2020;71(15):841-843.

5. Republic of Turkey Ministry of Health General Directorate of Public Health. Study Guide and Infection Control Measures in Health Institutions in the Covid-19 Pandemic. Scientific Advisory Board Study. Ankara. 07.09.2020. https://covid19bilgi.saglik.gov.tr/tr/covid-19.

6. Republic of Turkey Ministry of Health General Directorate of Public Health. Dental Procedures to be followed during the Covid-19 Outbreak. Scientific Advisory Board Study. Ankara. 04.16.2020. https://covid19bilgi.saglik.gov.tr.

7. CDC. Interim Infection Prevention and Control Guidance for Dental Settings During the COVID19 Response. Guidance for Dental Settings. May 19. https://www.cdc.gov/coronavirus/2019ncov/hcp /dental-settings.html.

8. Amato A, Caggiano M , Amato M , Moccia G , Capunzo M , Decaro CF: Infection Control in Dental Practice During the COVID-19 Pandemic. Int J Environ Res Public Health. 2020 17(13):4769-4780 .

9. Jakubovics N., Greenwood M., \& Meechan J.G: General medicine and surgery for dental practitioners: part 4. Infections and infection control. British Dental Journal. 2014;217(2):7377.

10. World Health Organization, Laboratory biosafety guidance related to coronavirus disease 2019(COVID-19):interim guidance,12 February 2020 https://www.who.int/emergencies/diseases/ novel- coronavirus-2019.

11. Papi P,Murro BD,Penna D,Pompa G: Digital prosthetic workflow during COVID-19 pandemic to limit infection risk in dental practice. Oral Disease 2020;00:1-4. 
12. Sivaramakrishnan G., Alsobaiei M, Sridharan K: Patient preference and operating time for digital versus conventional impressions: a network meta-analysis. Australian Dental Journa 2020; 65, 58-69.

13. Williams DW, Chamary N, Lewis MA, Milward PJ, McAndrew R: Microbial contamination of removable prosthodontic appliances from laboratories and impact of clinical storage. $\mathrm{Br}$ Dent 2011;211(4):163-6.

14. Chidambaranathan A, Balasubramanium M: Comprehensive Review and Comparison of the Disinfection Techniques Currently Available in the Literature. Journal of Prosthodontic 2019; 28(2):849-856.

15. Mortadi NA, Al- Khatib A, Alzoubi K, Khabour OF: Disinfection of Dental Impressions: Knowledge and Practice Among Dental TeJ Family Med Prim Care. 2020 9(2): 793-797. Clin Cosmet Investig Dent. 2019;11:103-108 .

16. Hemalatha.R, Ganapathy D: Disinfection of Dental Impression- A Current Overview. J. Pharm. Sci \& Res. 2016; 8(7), 661-664.

17. Barenghi, L., Barenghi, A., Cadeo, C., \& Di Blasio, A: Innovation by Computer-Aided Design/Computer-Aided Manufacturing Technology: A Look at Infection Prevention in Dental Settings. Hindawi Biomed Research International. 2019; 1-15, Article ID 6092018. https://doi.org/10.1155/2019/6092018.

18. Al Zain S: Effect of chemical, microwave irradiation, steam autoclave, ultraviolet light radiation, ozone and electrolyzed oxidizing water disinfection on properties of impression materials: A systematic review and meta-analysis study. Saudi Dent J. 2020;32(4): 161-170.

19. Shikh A, Milosevic A: Effectiveness of Alcohol and Aldehyde Spray Disinfectants on Dental Impressions. Clin Cosmet Investig Dent. 2020; 12: $25-30$. 